\title{
Comparison of Physical and Mental Practice on Acquisition of Basketball Free Shot
}

\author{
Öner Gülbahçe \\ Correspondence: Öner Gülbahçe, Atatürk University, Kazım Karabekir Education Faculty, Erzurum, Turkey.
}

Received: November 26, 2018

doi:10.11114/jets.v6i12a.3886

\author{
Accepted: December 13, 2018 \\ Online Published: December 17, 2018 \\ URL: https://doi.org/10.11114/jets.v6i12a.3886
}

\begin{abstract}
The purpose of this research was to compare mental and physical practice on acquisition of basketball free shot. Forty inexperienced boys were selected randomly by a revised questionnaire of motor imagery-making of Hall and Martin (1997). They were divided into 2 groups; an experimental group and a control one. Group one carried out the whole practice sessions practically. Group two carried out the whole sessions regarding to the mental practice being achieved as internal imagery. The results showed that the mental practices have significant impact on acquisition of basketball free shot.
\end{abstract}

Keywords: mental, physical, practice, acquisition

\section{Introduction}

In spite of the mental exercises discovery and its effective methods in developing and recovering the motor learning, the new findings are being represented in this relation and various approaches of mental practices have been still developing in this regard. The carried out studies of the recent decades have shown that the mental practice is very similar to the practical exercise leading to the progression of motor skills (Reynar, 2015). In the other hand, they specified that the mechanisms being participated in learning process with high intensity physical exercise become more active during the mental practice (Maxwell, 2013). Deriskll (2014) has shown that the mental practice is as effective as the practical practice in learning of motor skills. But another group emphasized that the role of mental practice in motor learning is lower than practical exercise (Dogan et al, 2014). Some other researchers believe that the mental practice does not influence on motor learning (Murphy, 2006).). In this relation, Hall et al. (2013) believes that the lack of mental practice impact on motor learning is coming from the fulfillment of wrong mental practice. He also believes that a wrong imagination of doing an activity for the positive impacts can make negative consequences. The imagery-making action is that the person's actions will be planned in a serious way of the actions. The frequency of the motor actions should be totally recognized before responding to the physical need (Dogan et al, 2014). An interruption being achieved by a gymnast during the jumping is a good instance of the related theory. The interruption time is different than the type of jump; the jumper is reviewing his mind before the action of jumping trying to select the best time of the related jump. In relation to the benefit of the imagery-making issue, there have been many different agreements but they are mostly suspicious in this regard. Hence, the mental imagery-making issue is a complete brain cognitive affair having an intriguing role in increasing of the muscles' power (Reynar, 2015). The experimental documents have been shown in relation to mental imagery- making affairs regarding to basketball, volleyball, football and tennis. Also, there have been carried out some researches about the influence of the mental imagery-making practices to facilitate the rate of learning of sport and motor skills; however, the application of the related process has not been recognized in sport skills yet. Thus, the present study is to evaluate the practical practices and mental practices as an internal imagery-making case being achieved on the basketball free shot skill and performance. This study tries to respond the question whether the function of basketball free shot can be achieved in a group-based case or no? And is there any significant difference between these related groups? Can it be recommended to the coaches? Enisler et al (2015) also showed that the mental practice had a positive influence on the performance but its benefit had also long distance during the mental practice due to the related intervention in this regard. Maxwell (2013) showed that the physical and mental practices are similar together. However, the application of practice method should be followed with mental practice along with the same physical practice potentially. It also is a very expensive method in teaching. 


\section{Materials and Methods}

Due to the nature of the research subject, it is a semi- experimental research. This research included two experimental groups as following:

1- Group one carried out the whole practice sessions practically

2- Group two carried out the whole sessions regarding to the mental practice being achieved as internal imagerymaking

After the arrangement of related groups, acquisition test was carried out in order to assess the status of the entire subjects in before and after practice times.

\section{Data collection instrument}

The questionnaire of MIQR and personal background (age, height and weight), medical history (background of pain, surgery, pain in shoulders and elbow, wrist and damage in related muscles regarding to grasping and neurological and muscular diseases) and the sport history were applied in the study.

\section{Statistical community, volume of the sample, sampling method and data analysis method}

The statistical sample of the present study is based on Morgan table of three percent by the number of 40 boy students of Erzurum high schools were selected in this study. Also, the whole subjects were right-handed; the whole participants completed the related questionnaires as well as their sport background in this path. In the next step the ability of imagery- making of the whole people using a revised questionnaire of motor imagery-making of Hall and Martin (1997) were also evaluated that the whole boy participants were also randomly divided into two groups of practical (20 ones) and mental ( 20 ones).

\section{Data collection instrument and method}

After the early selection of the statistical sample, in the first step the ability of imagery-making of the whole people using MIQR questionnaire was evaluated. People without the related ability were eliminated from the sample; also, due to the skill being considered as the criteria of the study, the personal traits of the people were also eliminated from the sample because of their skill. After complete specifying and displaying of the basketball free shot skill as well as early explanation, the pre-test of basketball free shot was considered as the criteria of the study. This also includes 10 basketball free shot based on AAPHRED test and the score of the subjects was registered in 10 struggles. In continue, due to the necessary trainings, the related group will achieve the skill criteria for four weeks including three sessions by number of 30 iterations. For the reason, practical group achieves their practices as practically; the mental group will achieve their actions as internal imagery-making and the whole participants were asked to complete the related actions as internal showing their excitements practically as if they play in a real basketball court. In the end of the week, the related criteria skill was evaluated in order to check the function in this case. In the end of the fourth week, a similar pre-test was achieved. Three days later of the last practice, acquisition test was completed and the score of the subjects were registered in a list.

\section{Statistical method}

Due to the consideration of the parametric statistical hypotheses, $T$ independent test was used to find differences between related groups at $\mathrm{p}<-0.05$ level. The software SPSS20 was also used to calculate the raw data.

\section{Results}

Table 1. Descriptive statistical indices regarding to mental practice and practical practice in various steps of pre- test and acquisition tests

\begin{tabular}{lllccc}
\hline Groups & & $\mathrm{N}$ & $\mathrm{M}$ & Std dev. & Err. \\
\hline Practical & Pre-test & 20 & 6.58 & 2.45 & 0.45 \\
& Acquisition & 20 & 11.23 & 2.58 & 0.49 \\
Mental & Pre-test & 20 & 6.95 & 2.76 & 0.43 \\
& Acquisition & 20 & 9.12 & 2.36 & 0.46 \\
\hline
\end{tabular}

Table 1 represents the mean comparison of pre-test, acquisition test of basketball free shot scores in both groups

Table 2. Results of mental practices significance on acquisition of basketball free shot of participants

\begin{tabular}{|c|c|c|c|c|c|}
\hline Statistical indices/groups & $\mathrm{N}$ & M St. & $\mathrm{df}$ & $\mathrm{T}$ & $\mathrm{P}$ \\
\hline Mental practice & 20 & 0.41 & 38 & 1.11 & $0.25^{*}$ \\
\hline Practical practice & 20 & 1.05 & & & \\
\hline
\end{tabular}


Table 2 shows that the mental practices have positive impact on the acquisition of subjects basketball free shot.

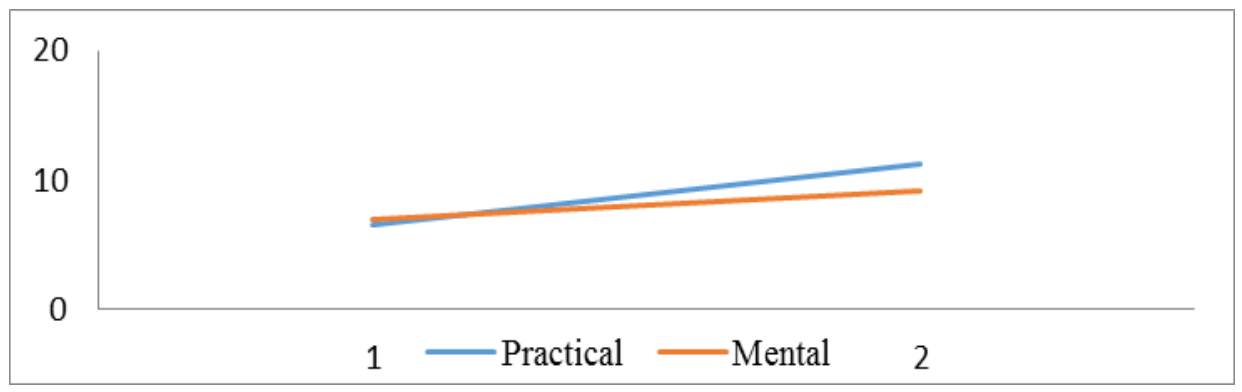

Figure. Mental and practical differences

\section{Discussion and Conclusion}

As it shown in findings of the research, the mental practices have significant impact on the acquisition of basketball free shot of the under study people with control group. In other words, in addition to the practical practices, it is expected that it can be effective on the motor skills learning process. The mental practice can be progressive on the subjects' acquisition criteria skill elements. Maxwell (2013), Hall et al (2013), Deriskll (2014), Cakmaci (2016) confirmed the present study results potentially. In other researches, it is shown that the mental practice can progress the motor learning skills. However, there have been other studies that they have also shown that mental practice does not have an impact on the motor skills. In addition, Dogan et al (2014) in the study of effectiveness of mental and practical practices concluded that the mental practice does not enough impact on the performance of the under study people.

Jackson et al (2014) found that mental practice has an impact on a new task of motor learning. He concluded that the mental practice has an equal impact on skill of throwing relation to automatic system changes in imagery- making of an activity. As it shown, these results support the present study results fairly in this regard. It seems logical that some combinative methods could be beneficent so that an athlete can feel of having enough benefits of the same method in this pavement (Reynar, 2015).

Indeed, the physical and mental practice combination is not better than only physical practice in an equal duration. In the other hand, many studies led by Maxwell (2013) showed that the subjects practicing mental practice have lower recovery in compare to subjects in physical practice group. But in compare to the control group without having any practices showed better mental practice in this case. Deriskll (2014) observed that the mental practice is effective in throwing but the physical practice had effective efficacy in this regard. Epstein (2013) in a two mega-analysis studies carried out on the imagery- making case concluded that the performance is appeared highly efficient in mental practice particularly in cognitive tasks in compare to motor tasks (Epstein, 2013)). In this research the motor skill (basketball free shot) is used that some of the results of the research were affected on the criteria skill. The experimentation of Ryan and Simon (1982) made another observation supporting the foundation of the effectiveness of mental practice case. The researchers concluded that if the mental practice is basically a cognitive issue, it should be effective in learning skills. In meta-analysis studies led by Epstein (2013), it is shown that the mental practice is very effective on the motor and cognitive actions but its effectiveness is higher on the cognitive activities learning process. In this research the novice subject was used in completing the criteria skill (basketball free shot) and some researchers believe that there is a reverse relationship between the experience level and mental practice effectiveness. Along this, Sanders et al (2016) showed that the mental practice has the highest impact in early steps (verbalcognitive step) and there is a reverse relationship between the experience level and the effectiveness of mental practice. As it observed, the results of the studies support the effectiveness of the mental practice. However, some other research results do not support the same idea in this case so that Cakmaci (2016) in a study observed the impact of mental imagery-making on the performance of skillful and novice football players equally. In another research led by Jackson et al (2014) this hypothesis is fairly rejected because the researchers found that the mental practice does not have an impact on the learning of new motor task. In turn, people having little familiarity with this case benefited of the mental and physical practices in this regard. Generally, the results of the research are coincident with the research of Deriskll (2014). As it shown from the results of the present study, there is no significant difference between the acquisition scores and learning groups of mental and practical issues among boys and girls; the results of the research is coincident with the result of Reynar's research. Reynar (2015) compared the mental practice on men and women and found that the men and women benefit equally of their mental imaginations during a routine educational plan. In relation to the made controversy, it can be pointed to the degree of learning impact through the mental practice in this field. The ability of imagery- making is a great impact on the mental practice. The studies 
showed that people with low imagery-making ability cannot progress in relation to their motor skill performance and vice versa. The practice duration is another effective factor in mental practice issue. As it shown before, the related factors can lead the mental practice to have many various impacts in motor skills. In this research, the external imagery-making is used that some researchers have been confirmed it as well. This idea has been designed by Jacobsen and Shaw. They found that the external imagery-making increases the muscular activity in compare to internal imagery issue. Due to these results, Epstein (2013) carried out a research in relation to the motor learning in internal imagery-making case that the results were equal together wonderfully. Reynar (2015) in comparison of internal and external imagery-making showed that the external imagery- making has the highest impact in the motor patterns. It also is relied on timing and periodical arrangement. The internal imagery-making is not roughly effective in motor skills. Since the mental practice is coming from the feedback of motor sensory deeply, hence the internal and external imagery-making issues make higher levels because these types of imagery-making affairs are coming along with higher motivational and muscular activities.

\section{References}

Cakmaci, A. (2016). Study and comparison of mental practice impacts as a supplementary method for practical exercise and learning of basketball shooting skill among boy and girl students. Surgical skills by medical students, 191(5), 1811-1814.

Deriskll, J. E., Copper, C., \& Morgan. A. (2014). Does mental practice enhance performance, Journalof applied psychology (JAP), 79, 481-492.

Dogan, P. L., Doyon, J., Richards, C. L., \& Malouin, F. (2014). The efficacy of combined physical and mental practice in, the learning of a foot -sequence task after stork: A case report, Neurorehabil, neural, repair, 18(2), 106-111.

Enisler, J. E., Copper, C., \& Morgan, A. (2015). Does mental practice enhance performance, Journal of applied psychology, 79, 481- 492.

Epstein, M. L. (2013). The relationship of mental imagery and mental rehearsal to performance of motor task, Journal of sport psychology, 2, 211-220.

Hall, C. R., \& Martin, K. A. (1997). Measuring movement imagery abilities: A revision of the Movement Imagery Questionnaire. Journal of Mental Imagery, 21, 143-154.

Hall, G. R., Buckalz, E., \& Fishburne, G. J. (2013). Imagery and the Acquisition of Motor Skills. Canadian Journal of Sport Sciences, 17(1), 19-27.

Hall, J. C. (2010). Imagery practice and the development of surgical skills, Am Journal surgical, 184(5), 465-470.

Jackson, P. L., Doyon, J., Richards, C. L., \& Malouin, F. (2014). The efficacy of combined physical and mental practice in the learning of a foot -sequence task after stork: A case report, Neurorehabil, 18(2), 106-111.

Maxwell, M. (2013). Imagery-making psychology, Shabahang publication

Murphy, L. (2006). Effect of a cognitive specific imagery intervention on the soccer skill performance of young athletes aged 11-12 and 13-14, Journal of Sport Psychology, 121-128.

Reynar, M. (2015). Sport Psychology, Olympic national committee publication. Erdal Publication.

Ryan, E. D., \& Simons, J. (1982). Efficacy of mental imagery in enhancing mental rehearsal of motor skills, Journal of Sport Psychology, 4, 41-51.

Sanders, C. W., Sadoski, M., \& Bramson, R. (2016). Comparing the effects of physical practice and mental imagery rehearsal on learning basic surgical skills by medical students, American Journal of Obstet Gynecol., 191(5), 1811-1184.

\section{Copyrights}

Copyright for this article is retained by the author(s), with first publication rights granted to the journal.

This is an open-access article distributed under the terms and conditions of the Creative Commons Attribution license which permits unrestricted use, distribution, and reproduction in any medium, provided the original work is properly cited. 